Karen K. Lindfors, MD

John M. Boone, PhD

Thomas R. Nelson, PhD

Kai Yang, MS

Alexander L. C. Kwan, PhD²

DeWitt F. Miller, BE
${ }^{1}$ From the Department of Radiology, University of California, Davis Medical Center, Suite 3100, 4860 Y St, Sacramento, CA 95817 (K.K.L., J.M.B., K.Y., A.L.C.K., D.F.M.); and the Department of Radiology, University of California, San Diego, La Jolla, Calif (T.R.N.). Received March 1, 2007; revision requested May 7; revision received July 18; accepted August 16; final version accepted September 25. Supported by grant EB002138 from the National Institute of Biomedical Imaging and Bioengineering. Address correspondence to K.K.L. (e-mail: kklindfors @ucdavis.edu).

${ }^{2}$ Current address: Department of Radiology and Diagnostic Imaging, University of Alberta, Edmonton, Alberta, Canada.

\title{
Dedicated Breast CT: Initial Clinical
} Experience $^{1}$

Purpose:

Materials and Methods:

Results:

Conclusion:
To prospectively and intraindividually compare dedicated breast computed tomographic (CT) images with screenfilm mammograms.

All patient studies were performed according to protocols approved by the institutional review board and Radiation Use Committee; informed consent was obtained. A breast CT scanner prototype was used to individually scan uncompressed breasts in 10 healthy volunteers (mean age, 52.1 years) and 69 women with Breast Imaging Reporting and Data System category 4 and 5 lesions (mean age, 54.4 years). In women with lesions, breast CT images were compared with screen-film mammograms by an experienced mammographer and ranked with a continuous scale of 1-10 (score 1, excellent lesion visualization with CT and poor visualization with mammography; score 5.5, equal visualization with both modalities; and score 10 , poor visualization with CT and excellent visualization with mammography). A Wilcoxon signed rank procedure was used to test the null hypothesis that ratings were symmetric at about a score of 5.5 for the entire group and for distinguishing microcalcifications versus masses and other findings and benign versus malignant lesions and for effect of breast density on lesion visualization. Women were asked to compare their comfort during CT with that during mammography on a continuous scale of 1-10. With a Wilcoxon signed rank procedure, the null hypothesis that comfort ratings were symmetric about a score of 5.5 (equal comfort with CT and mammography) was tested.

Overall, CT was equal to mammography for visualization of breast lesions. Breast CT was significantly better than mammography for visualization of masses $(P=.002)$; mammography outperformed CT for visualization of microcalcifications $(P=.006)$. No significant differences between CT and mammography were seen among benign versus malignant lesions or for effect of breast density on lesion visualization. Subjects found CT significantly more comfortable than mammography $(P<.001)$.

Some technical challenges remain, but breast CT is promising and may have potential clinical applications.

() RSNA, 2008 
B reast cancer screening by using screen-film mammography has led to a substantial reduction in mortality (1); however, all cancers are not detected with screen-film mammography $(2,3)$. Evidence has shown that digital mammography outperforms screen-film mammography for women with denser breast tissue (4). Despite the improvement in screening accuracy with the use of digital mammography compared with screen-film mammography, overlying normal breast tissue can still obscure a breast lesion and hinder detection and/or diagnosis. Digital breast tomosynthesis (5), a form of limited-angle tomography, has been developed as one approach to improve the accuracy of breast cancer screening. Contrast material-enhanced magnetic resonance (MR) imaging, with its multiplanar imaging capabilities, is being used to screen women at high risk for breast cancer $(6,7)$ and also for other specific diagnostic situations, but it is expensive and access is limited. In our laboratory, we have developed a dedicated breast computed tomographic (CT) system capable of cone-beam CT of the breast. This system provides full tomographic imaging of the breast for

\section{Advances in Knowledge}

- Overall, breast CT images were approximately equal to screenfilm mammograms for visualization of breast lesions.

- Breast CT images were significantly better than screen-film mammograms for visualization of masses $(P=.002)$, but screenfilm mammograms were significantly better than breast CT images for visualization of microcalcification lesions $(P=.006)$; there were no significant differences in the two types of images for distinguishing benign versus malignant lesions or for effect of breast density on lesion visualization.

- Women found breast CT to be significantly $(P<.001)$ more comfortable than screen-film mammography. either screening or diagnostic breast examination.

The concept of breast CT was studied in the 1970s in the early years of CT technology development (8-12). The system produced CT images of the breast, which was immersed in a water bath, with voxels measuring $1.53 \times$ $1.53 \times 10 \mathrm{~mm}$. The voxel volume of the cone-beam breast scanner developed at our institution is 2500 times smaller than the early prototype system of a generation ago. Other investigators (13-15) are studying the potential of breast CT by using cone-beam detector systems, but to our knowledge, there have been no clinical series yet reported. Thus, the purpose of our study was to prospectively and intraindividually compare dedicated breast CT images with screen-film mammograms.

\section{Materials and Methods}

\section{Technical Aspects of Dedicated Breast CT}

The breast CT scanner prototype was designed at the University of California, Davis, Sacramento, Calif, by using offthe-shelf components where possible. A water-cooled tungsten anode $\mathrm{x}$-ray tube (Comet, Flamatt, Switzerland) was incorporated into the scanner, with a $0.40 \times 0.40-\mathrm{mm}$ focal spot and $0.30 \mathrm{~mm}$ of added copper filtration. A flat-panel X-ray detector (Paxscan 4030CB; Varian Imaging Systems, Palo Alto, Calif) that was $40 \mathrm{~cm}$ wide and $30 \mathrm{~cm}$ tall was used, and this cesium iodide indirect detector bins $2 \times 2$ pixels to achieve 30 -frame per second acquisition rates at a pixel matrix of $1024 \times 768$, resulting in $0.388-\mathrm{mm}$ pixel dimensions at the detector. Projected back to the CT

\section{Implication for Patient Care}

- Dedicated breast CT is currently investigational but may eventually have applications in screening or diagnostic evaluation for breast cancer, as a more accessible replacement for breast MR imaging or as a guidance method for robotic breast biopsy or tumor ablation procedures. scanner isocenter as is the norm in CT, the detector elements measure approximately $0.208 \mathrm{~mm}$. The gantry rotates in the horizontal plane and is powered by an integrated bearing-motor-encoder system (Kollmorgen, Radford, Va).

The commercially available components described previously were integrated into the design of a pendant-geometry breast CT scanner (Fig 1). A local machine shop was used to fabricate the individual components for the frame, tabletop, gantry, and other components, and the breast CT scanner was fabricated in our laboratory. After computer integration, the system was fully characterized in terms of its image quality performance.

Each breast is scanned individually without compression in the pendant position while the patient lies prone on the scanning table. The scan field of view measures $21 \mathrm{~cm}$ in diameter. At 30 frames per second, 500 cone-beam projection images are acquired $360^{\circ}$ around the patient's breast in $16.6 \mathrm{sec}$ onds. The breast CT technique factors (tube voltage and tube current-time product) were calculated to deliver the same mean glandular radiation dose to the breast as does two-view mammography, on the basis of Monte Carlo analyses performed for this purpose (16). Women with larger and denser breasts receive a greater radiation dose in

Published online before print 10.1148/radiol.2463070410

Radiology 2008; 246:725-733

\section{Abbreviations:}

BI-RADS = Breast Imaging Reporting and Data System

DCIS = ductal carcinoma in situ

IDC = invasive ductal carcinoma

\section{Author contributions:}

Guarantor of integrity of entire study, K.K.L.; study concepts/study design or data acquisition or data analysis/ interpretation, all authors; manuscript drafting or manuscript revision for important intellectual content, all authors; manuscript final version approval, all authors; literature research, K.K.L., J.M.B.; clinical studies, all authors; experimental studies, K.K.L., J.M.B., T.R.N., K.Y., A.L.C.K.; statistical analysis, K.K.L., J.M.B., K.Y., D.F.M.; and manuscript editing, K.K.L., J.M.B., T.R.N., K.Y., D.F.M.

Authors stated no financial relationship to disclose. 
mammography, and the technique factors in the breast CT scanner are also increased for larger and denser breasts in order to keep $\mathrm{x}$-ray quantum noise to a reasonable level. The scanner has been used almost exclusively at $80 \mathrm{kVp}$, with tube current-time product values from 50 to $120 \mathrm{mAs}$, depending on breast size. For patients with smaller breasts $(12-\mathrm{cm}$ breast diameter at the chest wall), the mean glandular dose was approximately $2.5 \mathrm{mGy}$; for patients with typical breast size $(14-\mathrm{cm}$ breast diameter at the chest wall), the mean glandular dose was approximately $6.0 \mathrm{mGy}$; and for women with larger breasts (16-cm breast diameter at the chest wall), the mean glandular dose was about $10.3 \mathrm{mGy}$.

The projection images are used to reconstruct $300-500512 \times 512$ images. The native reconstructed CT images are produced in the coronal plane, but the complete volume data set can be used to produce tomographic images in any offaxis orientation. Breast CT images for our study were viewed in stack mode (sequentially) at a flat-panel liquid crystal display monitor in the coronal, sagittal, and transverse planes by using software developed specifically for breast CT. Contrast and brightness could be manipulated for optimal viewing, and a zoom function was available. The section width thickness could also be adjusted.

\section{Pilot and Clinical Studies}

Patient scanning began on November 22, 2004, following institutional review board approval. All patient studies were performed according to protocols approved by the institutional review board; informed consent was obtained from each participant. Because of the nature of this study, institutional review board approval required additional approval from the Radiation Use Committee and the Cancer Center Scientific Review Committee, both of which were obtained. The breast CT technique factors were adjusted to deliver a mean glandular dose to the breast comparable to that from each woman's two-view mammographic examination. The consent form described the risks associated with the additional radiation of the breast CT scans by using nomenclature defined by the institutional review board and Radiation Use Committee. The study was conducted in compliance with Health Insurance Portability and Accountability Act regulations.

A pilot study of 10 healthy volunteers (mean age, 52.1 years; range, 40.0-67.3 years) was performed to assess the quality of the breast CT images, the feasibility of a 16.6-second breath hold, breast positioning in the scanner, and the patient's level of comfort during the breast CT examination. Breast CT image quality and positioning were assessed by a mammographer (K.K.L., with more than 20 years of experience in breast imaging). Breast CT images were subjectively assessed for contrast, motion blur, and anatomic detail. The breast CT images were examined for centering within the scanner. All women were asymptomatic and had negative findings at screening mammography performed within 6 months prior to breast CT. Immediately after their breast CT study, women were asked to complete a questionnaire in regard to their comfort during breast CT. They were asked to rate the comfort of their position on the breast CT table (from 1 for poor to 10 for excellent), how difficult it was for them to hold their breath during the examination (from 1 for very difficult to 10 for not at all difficult), and their overall comfort level during breast CT (from 1 for very uncomfortable to 10 for extremely comfortable). Volunteers were also asked to compare their comfort level during breast CT with their comfort level during screen-film mammography (from 1 for much worse than screen-film mammography to 10 for much better than screen-film mammography). Additional comments in regard to their experience with breast CT were solicited in writing on the questionnaire.

For the clinical study of breast CT, 69 symptomatic and asymptomatic women (mean age, 54.4 years; range, 35.7-82.0 years) with Breast Imaging Reporting and Data System (BI-RADS) category 4 or 5 lesions after diagnostic breast imaging were asked to undergo bilateral unenhanced dedicated breast CT prior to core biopsy. Women with BI-RADS category 3 lesions who requested biopsy instead of short-interval follow-up were also eligible. All four of the mammographers at our institution (including K.K.L.) recruited these patients in a sequential manner. Recruitment occurred at the time the patient was given her diagnostic breast imaging results. Concordance between screenfilm mammography and breast CT was determined with lesion location, type, and size. Women who underwent breast CT were also asked to complete the comfort questionnaire mentioned previously at the conclusion of the examination.

To assess the conspicuity of abnormalities, breast CT images of the affected breast only were compared with conventional screen-film mammograms by using the craniocaudal and mediolateral oblique views only; no other images from diagnostic imaging studies were included in the comparison. Lesion conspicuity on breast CT images compared with that on screen-film mammograms was subjectively assigned a score by an experienced mammographer (K.K.L.) on a continuous scale from 1 to 10 , where 1 indicated excellent conspicuity on breast CT images and poor conspicuity on screen-film mammograms, 5.5 indicated equal conspicuity with both types of images, and 10 indicated poor conspicuity on breast CT images and excellent conspicuity on screen-film mammograms. The comparison was nonblinded, as this report describes our initial clinical experience. Breast CT was the initial study for which findings were interpreted for the comparison; if the

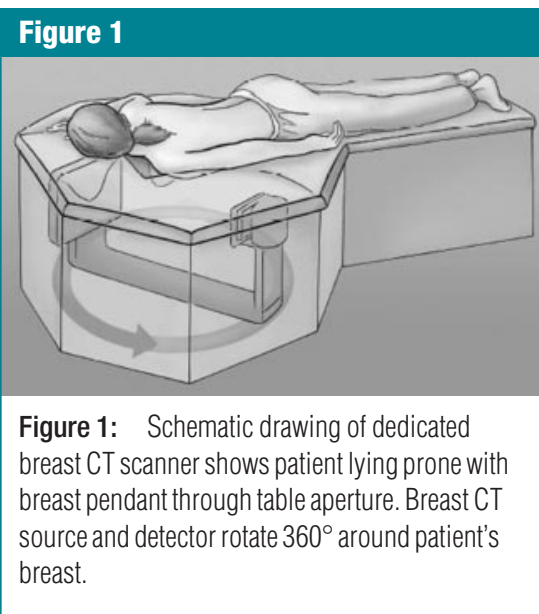


lesion was not identified on the breast CT image, the screen-film mammogram was examined and, with the knowledge of findings from it, the breast CT image was then reinterpreted for the comparison.

Women in the clinical study were categorized into a group with palpable breast masses and an asymptomatic group. Lesions were classified according to type (microcalcification lesions, masses, or other findings) and were correlated with the pathologic results from the core biopsies. Lesion size was calculated from the screen-film mammogram. Breast density (fatty replaced, scattered fibroglandular densities, heterogeneously dense, or dense) was evaluated by using the screen-film mammography report, and the number of types of lesions, classified according to breast density, was tabulated.

Results of the comparison of breast CT with screen-film mammography for lesion conspicuity were analyzed for the study group as a whole and also according to the type of lesion (microcalcification lesions vs other lesions) seen on the mammogram, the pathologic diagnosis (benign vs malignant lesions), and the type of breast density as described in the mammography report. Breast CT images were also examined for inclusion of posterior tissue, and the number of women in whom the pectoralis major muscle was visualized on a breast CT image was tabulated.

Patients were excluded from the comparison of breast CT with screen-film mammography if the breast CT image was judged to be of poor technical quality because of motion blurring or the lesion was out of the field of view on the breast CT image. Breast CT studies were not repeated in these women. These women were excluded from the study because lesion conspicuity on breast CT images could not be assessed.

A study of contrast-enhanced breast CT in four patients with BI-RADS category 5 mass lesions who were randomly selected and who consented to contrast agent administration was also performed. One hundred milliliters of iodixanol injection, $320 \mathrm{mg}$ of iodine per milliliter (Visipaque 320; GE Healthcare, Princeton,
$\mathrm{NJ}$ ), was injected intravenously by using a power injector (Mark V Plus; Medrad, Pittsburgh, Pa) during 25 seconds, and breast CT was performed after a 90-140second delay. Contrast-enhanced breast CT images were subjectively compared with mammograms (K.K.L.). Only one of these women underwent unenhanced breast CT, and that examination was also included in the clinical study comparison, as described before; the other three women who underwent contrast-enhanced breast CT only were not included in the clinical study comparing unenhanced breast CT with screen-film mammography. All four women who underwent contrast-enhanced breast CT completed the comfort questionnaire.

\section{Statistical Analysis}

The quantitative data resulting from the radiologist's comparison between breast CT and screen-film mammography (overall and according to type of lesion, pathologic diagnosis, and type of breast density) and the patient ratings for comfort of the procedure were summarized descriptively by using univariate statistics (mean, median, standard deviation, range). We then calculated the 95\% confidence intervals for the median rating by using a bootstrap procedure (17). For questions that asked the patient or the radiologist to compare breast CT with screen-film mammography, a rating of 5.5 on a scale of 1 (for strongly prefer mammography or mammography was much better) to 10 (for strongly prefer CT or CT was much better) would correspond to a rating of no preference. For these questions, we tested the null hypothesis that the ratings were symmetric about 5.5 by using a Wilcoxon signed rank procedure. All statistical analyses were performed by using software (SAS/STAT; SAS Institute, Cary, NC), and all tests were two sided. Significance was defined as a difference with $P<.05$.

\section{Results}

\section{Pilot Study}

Breast CT scans in nine of 10 healthy volunteers demonstrated excellent ana- tomic detail and contrast. Motion blurring was seen on a scan in one patient. All volunteers were able to sustain the 16.6-second breath hold. There were no problems with breast positioning or patient comfort.

\section{Clinical Study}

Sixty-nine women with BI-RADS category 4 or 5 breast lesions underwent unenhanced breast CT prior to core biopsy. Four of these women were excluded from the study analysis: Two were excluded because of movement during breast CT (possibly related to the breath hold). Two others were excluded because the mammographically visible lesions were not in the field of view on the breast CT scan; one lesion was in the axillary tail and the other was in the anterior aspect of the breast in a large-breasted woman. Two women had bilateral breast lesions. The breast CT images were, therefore, compared with the screenfilm mammograms in a total of 67 breasts in 65 patients.

Forty-nine $(73 \%)$ of 67 breast lesions were not palpable at clinical breast examination. The distribution of the types of breast density, as described in the mammography reports, was as follows: fatty replaced, one $(1.5 \%)$ lesion; scattered fibroglandular densities, 27 (40.3\%) lesions; heterogeneously dense, 26 (38.8\%) lesions; and dense, $13(19.4 \%)$ lesions.

On the basis of the diagnostic breast imaging evaluation, there were $38 \mathrm{BI}$ RADS category 4 lesions, 28 BI-RADS category 5 lesions, and one BI-RADS category 3 lesion. There were 52 (78\%) masses, 12 (18\%) microcalcification lesions, and three (4\%) lesions of other types. In the group with dense breasts, there were four (31\%) microcalcification lesions, whereas in the women with heterogeneously dense breasts there were three $(12 \%)$ microcalcification lesions, and in those with scattered fibroglandular densities, there were four (15\%) such lesions. The patient with fatty-replaced breasts had a microcalcification lesion.

Fifty-eight (87\%) of 67 lesions were identified at breast CT (Figs 2-4). Two 
of the lesions identified at screen-film mammography but not seen at breast CT were later proved to represent summation artifacts and biopsy was not performed, so $89 \%$ (58 of 65) of true lesions seen at screen-film mammography were identified at breast CT. Among the seven true lesions not seen at breast CT, three were malignant; one was a 5-mm cluster of microcalcification lesions (ductal carcinoma in situ [DCIS]), one was an area of diffuse microcalcification lesions in dense tissue (DCIS), and one was a mammographically subtle $15-\mathrm{mm}$ mass that was best seen at ultrasonography (US) (invasive ductal carcinoma [IDC]). Among the benign true lesions that were not identified at breast CT, one was diabetic mastopathy, which was palpable but poorly visualized at screen-film mammography; one was a $2-\mathrm{mm}$ cluster of microcalcifications (lobular carcinoma in situ); one was a $3-\mathrm{cm}$ area of microcalcifications (fibrocystic change); and one was a 4-mm cyst (Table 1$)$.

In one patient (Fig 5), a small satellite cancer adjacent to the index lesion was imaged at breast CT. It had not been observed at screening or diagnostic mammography.

The mean size of the lesions at screen-film mammography was 13.9 $\mathrm{mm}$ (range, 2-60 $\mathrm{mm}$ ); the mean sizes of masses and microcalcification lesions were $13.75 \mathrm{~mm}$ and $14.54 \mathrm{~mm}$, respectively. The pectoralis major muscle was identified at breast CT in 12 (18\%) of 67 breasts.
In the comparison of breast CT with screen-film mammography, the overall mean lesion conspicuity score was 5.4 , indicating that visualization with breast CT was approximately equal to that with screen-film mammography, for 67 breasts (Table 2 ).

\section{Breast CT Comfort Questionnaire}

Results of the patient comfort survey including the 10 healthy volunteers, the 69 women scanned in the clinical study, and the three additional women who underwent contrast-enhanced

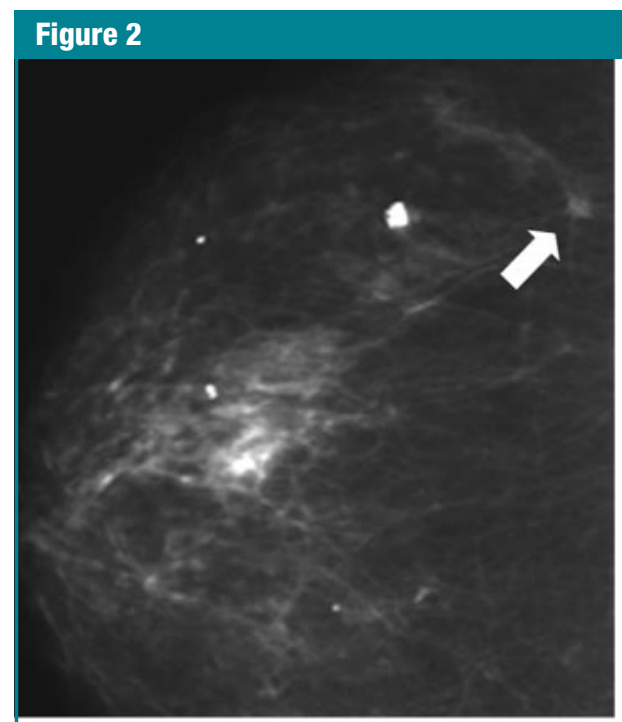

a.

Figure 2: (a) Craniocaudal screen-film mammogram of right breast shows 4-mm IDC (arrow). (b) Coronal CT scan in same woman in same breast shows 4-mm IDC at 12-o'clock position (arrow). Lesion conspicuity score was 3 , indicating better lesion visualization at breast CT. breast CT only $(n=82)$ indicated that many women found it difficult to arch forward into the breast CT unit and that their necks were uncomfortable in this position or that the tabletop was too firm. When asked to rate breast CT comfort compared with screen-film mammography comfort, two-thirds of women gave a response of 10 on a scale of 1-10, indicating a very pronounced preference for breast CT $(P<.001$, Wilcoxon signed rank test for symmetric preference) (Table 3).

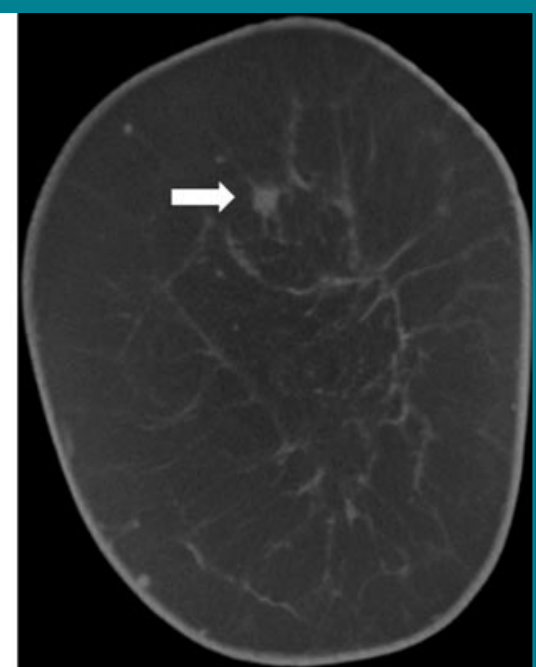

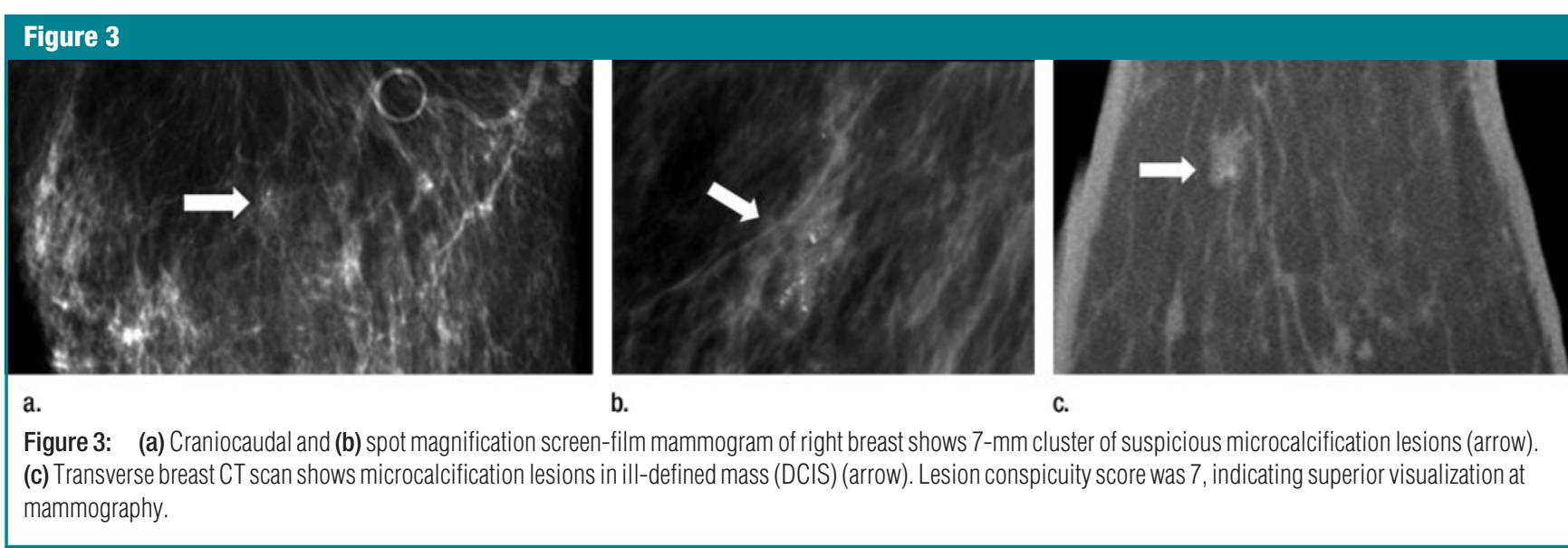




\section{Contrast-enhanced Breast CT}

Three of four patients who underwent contrast-enhanced breast CT proved to have IDC at pathologic examination. In one of those with IDC, a second lesion that had not been observed at standard breast imaging was identified near the chest wall (Fig 6). Another woman with IDC had diffuse DCIS with multiple areas of invasion; she had also undergone a core biopsy of a palpable area in the breast prior to breast CT. An area of enhancement was seen at breast CT, but comparison with the pathologic results was difficult because of the diffuse nature of her disease. The fourth woman who underwent contrast-enhanced breast CT had a benign lesion (sclerosing adenosis) with minimal enhancement.

\section{Discussion}

Initial clinical experience with our scanner prototype shows that overall breast CT performance is similar to that of screen-film mammography. Masses are

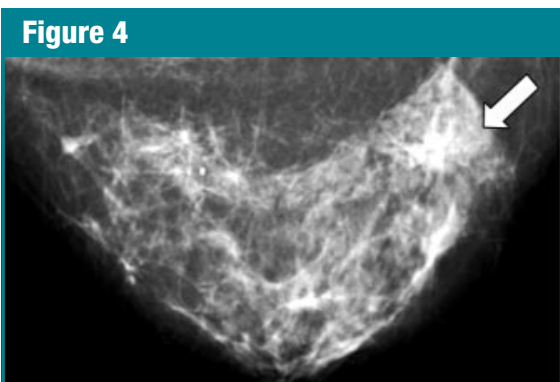

a.

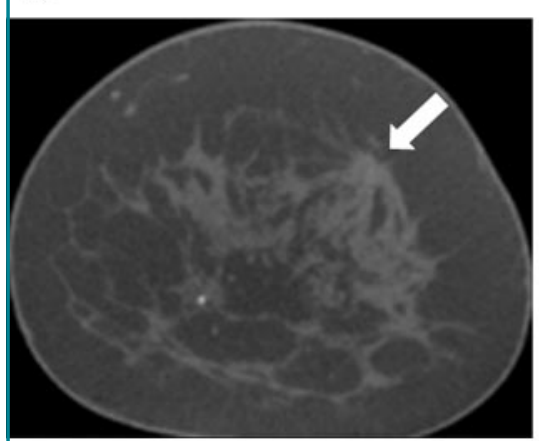

c.

Figure 4: (a) Craniocaudal screen-film mammogram and (b) transverse, (c) coronal, and (d) sagittal breast CT scans of left breast show a spiculated mass (IDC) (arrow) at 1-o'clock position in upper outer quadrant. Lesion conspicuity score was 5 , indicating slightly better visualization at breast CT.

b.

d. significantly more conspicuous on screen-film mammograms, but microcalcification lesions are not as well visualized on our early-generation dedicated breast CT images. There were no significant differences in the conspicuity of benign or malignant lesions with the two types of images. Breast density did not have a significant effect on lesion visualization on breast CT images, although women with dense breasts $(n=$ 13) tended to have lesions that were not as conspicuous on breast CT images as they were on screen-film mammograms. This difference may be caused by the higher percentage of microcalcification lesions, which are not as well visualized on breast CT images, in the group with dense breasts (31\%) as compared with the group with scattered fibroglandular densities $(15 \%)$ or heterogeneously dense breasts $(12 \%)$.

Women found breast CT to be significantly more comfortable than screenfilm mammography, probably because breast CT does not require compresbreast CT images compared with

sion. Current ergonomics of the breast CT tabletop, however, requires women to arch forward into the scanner in a position that some thought was uncomfortable. As refinements in design are made, it is likely that positioning can be made more comfortable.

There are many potential uses for breast CT. It may be useful in screening for breast cancer, although, to be applicable to the general population, visualization and characterization of microcalcification lesions will have to be improved. Our study was conducted at 80 $\mathrm{kVp}$, and this decision was based on a trade-off between patient dose and image noise. The contrast of microcalcification lesions will increase at lower tube voltage settings (or with different filtration levels), but a lower tube voltage at the same mean glandular dose to the breast will result in fewer detected $\mathrm{x}$-ray quanta and higher noise levels. The ultimate effect of tube voltage on signal-tonoise ratio is currently being studied.

Breast CT offers the advantage of three-dimensional anatomic detail and eliminates superimposition of glandular tissues. As a result, fewer women should
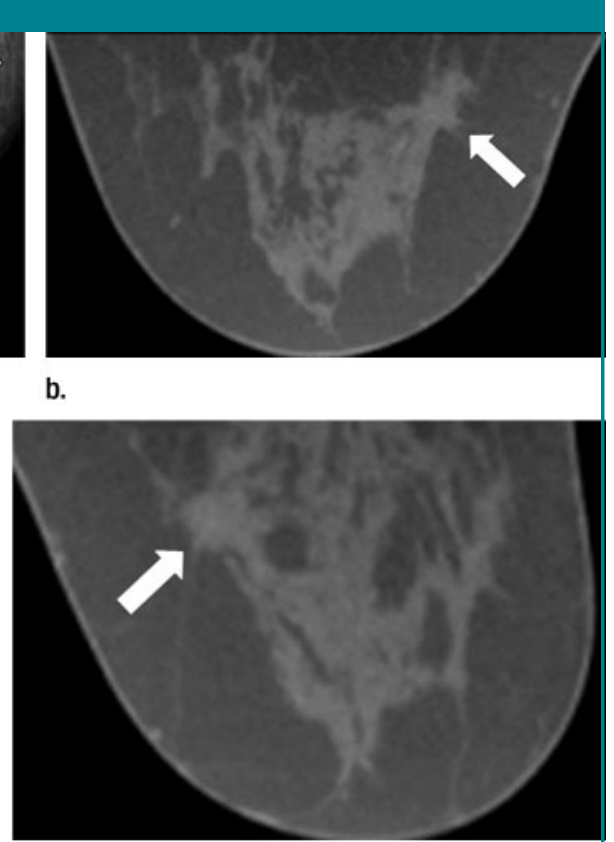

\begin{tabular}{|c|c|}
\hline \multicolumn{2}{|c|}{$\begin{array}{l}\text { Pathologic Diagnoses of Lesions } \\
\text { Identified in } 65 \text { Patients at Breast CT }\end{array}$} \\
\hline Diagnosis & No. of Patients ${ }^{*}$ \\
\hline \multicolumn{2}{|l|}{ Benign } \\
\hline Adenosis & $2(3)$ \\
\hline CAPSS & 2 (3) \\
\hline Fibrocystic change & $10(15)$ \\
\hline Fibroadenoma & $10(15)$ \\
\hline LCIS & $1(2)$ \\
\hline Other & $3(5)$ \\
\hline Total & $28(43)$ \\
\hline \multicolumn{2}{|l|}{ Malignant } \\
\hline DCIS & $8(12)$ \\
\hline IDC & $24(37)$ \\
\hline ILC & $1(2)$ \\
\hline IDC, DCIS & 1 (2) \\
\hline IDC, ILC & $2(3)$ \\
\hline Lymphoma & $1(2)$ \\
\hline Total & $37(57)$ \\
\hline
\end{tabular}

Note.-CAPSS $=$ columnar alteration with prominent apical snouts and secretions, ILC = invasive lobular carcinoma, LCIS = lobular carcinoma in situ.

* Numbers in parentheses are percentages. 
be recalled for evaluation of potential abnormalities that are summation artifacts; two lesions in our study that were seen at screen-film mammography but not at breast CT were later shown to be summation artifacts. It is also possible that the sensitivity of screening can be improved as a result of the capability to image a potential abnormality in multiple planes without overlapping tissue.

Breast CT may offer an alternative to MR imaging for screening high-risk women for breast cancer and for determining the local extent of disease in newly diagnosed breast cancers. It is likely that breast CT will be lower in cost than MR imaging, but the cost of breast CT is currently unknown. The examination time for breast CT is currently shorter than it is for MR imaging, but breast CT requires ionizing radiation. It is unknown whether contrast enhancement will be required for optimal performance of breast CT, but our preliminary work has shown that, in some cases, additional disease can be seen by using breast CT without contrast enhancement. As evidenced by the enhancement of the infiltrating cancers reported here, it is likely that malignant lesions will show patterns of enhancement that are similar to those seen at contrast-enhanced MR imaging (18) and at dual-energy subtraction mammography (19). Dual-energy high-tubevoltage subtraction projection images acquired with the breast CT scanner may be useful in assessing the kinetics of enhancement at far lower dose levels than are used to acquire breast CT images, and with good temporal sampling.

The breast CT scanner may provide an ideal imaging platform for development of robotic breast biopsy and tumor ablation devices. Stereotactic biopsy techniques rely on two stereo views for positioning, whereas breast CT provides a complete three-dimensional data set for guiding biopsy needle placement. Automated positioning techniques would make the biopsy procedure potentially more accurate, as well as faster and easier to perform, than it is with stereotactic or US guidance. In addition to using breast CT for biopsy needle guidance, there are a growing

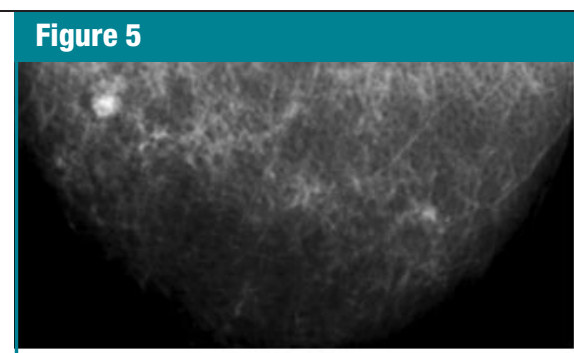

a.

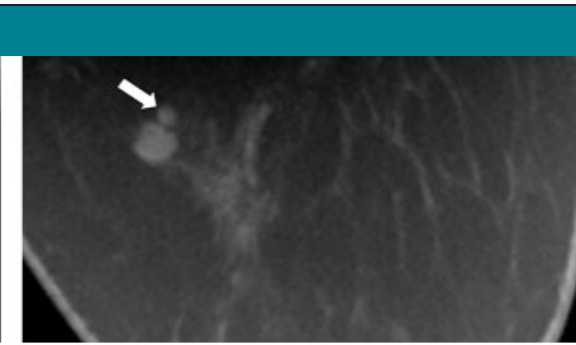

Figure 5: (a) Craniocaudal screen-film mammogram of right breast for comparison with (b) transverse breast CT scan, which shows satellite lesion (arrow), not observed on a, adjacent to index lesion (IDC). Lesion conspicuity score was 3 , indicating superior visualization at breast CT.

\section{Table 2}

Conspicuity of Lesions at Breast CT Compared with Screen-Film Mammography

\begin{tabular}{|c|c|c|c|c|}
\hline \multirow[b]{2}{*}{ Category } & \multirow{2}{*}{$\begin{array}{l}\text { No. of } \\
\text { Breasts }\end{array}$} & \multicolumn{2}{|c|}{ Score } & \multirow[b]{2}{*}{$P$ Value $^{\dagger}$} \\
\hline & & Mean \pm SD & Median* & \\
\hline All & 67 & $5.4 \pm 1.9$ & $5.5(5.0,6.0)$ & 48 \\
\hline \multicolumn{5}{|l|}{ Lesion type } \\
\hline Masses or other findings ${ }^{\ddagger}$ & 55 & $4.9 \pm 1.5$ & $5.0(4.6,5.4)$ & $.002^{\S}$ \\
\hline Microcalcifications & 12 & $7.8 \pm 1.9$ & $8.0(6.2,9.8)$ & $.006^{\S}$ \\
\hline \multicolumn{5}{|l|}{ Lesion diagnosis } \\
\hline Malignant & 37 & $5.7 \pm 1.9$ & $5.5(5.1,5.9)$ & .81 \\
\hline Benign & 28 & $5.3 \pm 2.0$ & $5.0(4.3,5.7)$ & .35 \\
\hline \multicolumn{5}{|l|}{ Breast density type } \\
\hline Fatty replaced & 1 & 9.0 & & \\
\hline Scattered fibroglandular & 27 & $5.1 \pm 1.4$ & $5.0(4.4,5.6)$ & .06 \\
\hline Heterogeneously dense & 26 & $5.3 \pm 1.8$ & $5.5(5.1,5.9)$ & .42 \\
\hline Dense & 13 & $6.4 \pm 2.8$ & $6.0(3.1,8.9)$ & .26 \\
\hline
\end{tabular}

Note.-Sixty-five patients were included in the analysis, but two had cancer in both breasts ( 67 breasts). A score of 5.5 indicates equal visualization with breast CT images and screen-film mammograms; a score of less than 5.5 indicates superior visualization with breast CT images; and a score greater than 5.5 indicates superior visualization with screen-film mammograms.

* Numbers in parentheses are the $95 \%$ confidence intervals.

${ }^{\dagger} P$ value was determined by using the Wilcoxon signed rank test with 5.5 (score for equal visualization with both types of images) subtracted.

₹ Includes 52 masses and three other findings.

$\S$ Significant.

number of therapeutic interventions that require accurate three-dimensional planning, guidance, and monitoring. Techniques such as radiofrequency ablation, cryoablation, or high-intensity focused ultrasound have found utility in other tumor sites, and it is possible that these minimally invasive approaches may offer alternatives to lumpectomy in the future. Such interventions will require an accurate method for determining tumor volume and the potential vol- ume of tissue destruction. Breast CT seems well suited for this application.

There are several immediate challenges that must be addressed in the continued development of breast CT. Interpretation time for radiologists is likely to be longer than it is for screenfilm mammography or digital mammography, but it may be shorter than it is for breast MR imaging. The additional time required for interpretation by the radiologist may be partially offset by the 
reduced number of recalled patients and the need for other diagnostic imaging that is expected with breast CT. Computer-aided detection with breast

\section{Table 3}

\section{Breast CT Comfort Survey Results in 82 Patients}

\begin{tabular}{llr} 
& \multicolumn{2}{c}{ Score } \\
\cline { 2 - 3 } Question & Mean \pm SD & Median* $^{*}$ \\
\hline Position $^{\dagger}$ & $6.7 \pm 2.6$ & $7(6.1,7.9)$ \\
Breath hold $^{\ddagger}$ & $7.7 \pm 2.3$ & $8(7.3,8.7)$ \\
Comfort $^{\S}$ & $7.9 \pm 2.1$ & $8(7.1,8.9)$ \\
Breast CT vs mammography" & $8.9 \pm 1.9$ & $10(10,10)$ \\
\hline
\end{tabular}

Note.-The number of patients includes 10 healthy volunteers, 69 patients with BI-RADS category 4 or 5 lesions determined at diagnostic breast imaging who underwent unenhanced breast CT before core biopsy, and three additional patients who underwent contrast-enhanced breast CT only. $P$ values were less than .001 for all questions and were determined by using the Wilcoxon signed rank test with 5.5 (score for equal visualization with both types of images) subtracted. SD = standard deviation.

* Numbers in parentheses are the $95 \%$ confidence intervals.

${ }^{\dagger}$ Position refers to comfort of the position on the breast CT table $(1=$ poor, $10=$ excellent).

${ }^{\ddagger}$ Breath hold refers to ease of 16.6 -second breath hold $(1=$ very difficult, $10=$ not at all difficult).

$\S$ Comfort refers to overall comfort of breast CT ( $1=$ very uncomfortable, $10=$ extremely comfortable).

"Breast CT versus mammography refers to comfort during breast CT as compared with comfort during screen-film mammography $(1=$ comfort was much worse than that of screen-film mammography, $5.5=$ comfort was equal to that of screen-film mammography, 10 = breast CT was much more comfortable than screen-film mammography).
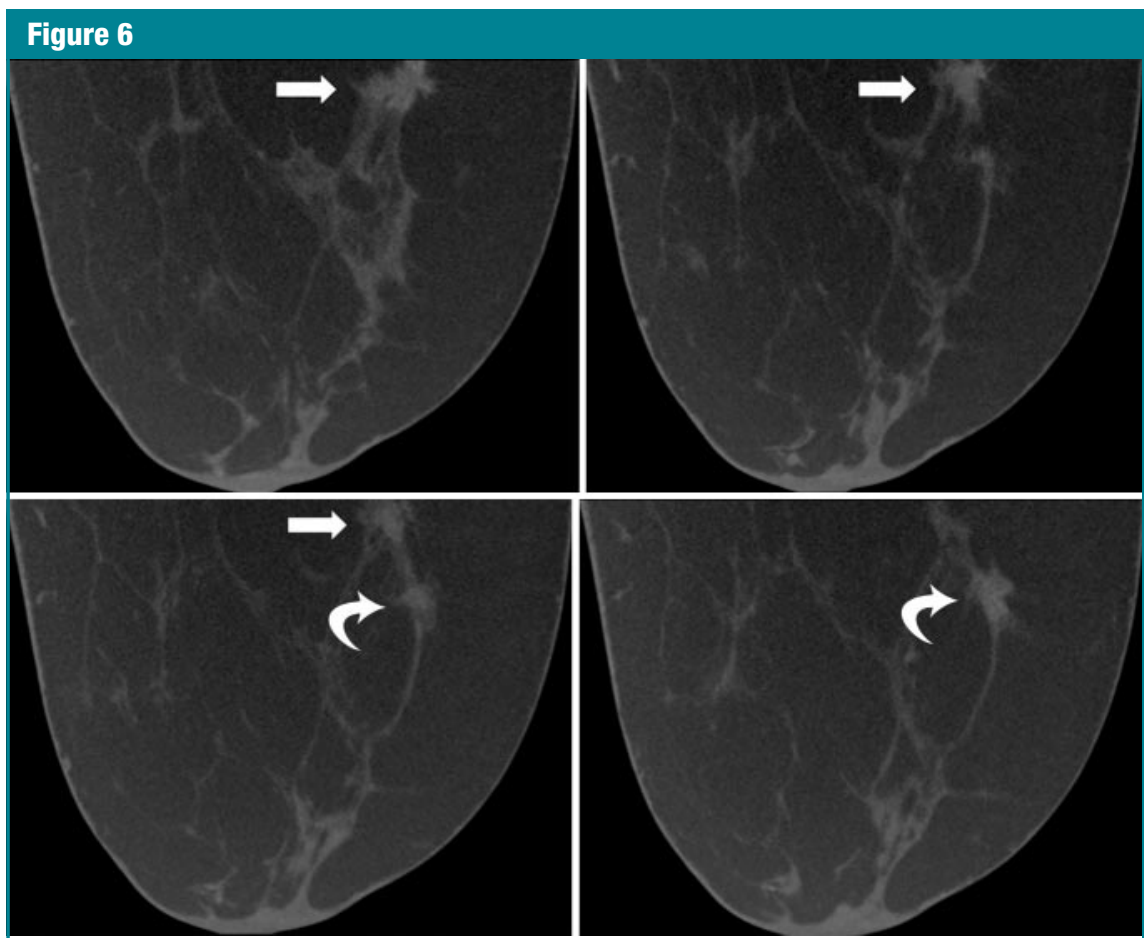

Figure 6: Sequential contrast-enhanced transverse breast CT scans show two enhancing, spiculated masses (arrows), both of which were IDC. Only posterior lesion (straight arrows) was seen on screen-film mammogram. The second anterior lesion (curved arrows) was not seen at mammography. musculature at breast CT. Visualization of the axillary tail of the breast also was limited with the scanner prototype used in this study. Refinements in the design of the breast CT table to allow increased visualization of the chest wall and axillary tail are under way and appear promising. In addition, the problem of anterior tissue being out of the field of view in large-breasted women, as in one of the excluded cases in our study, has been addressed by bolstering the breast so as to bring the anterior tissues closer to the chest wall in such women.

Two women were excluded from our study because of motion on the breast CT scan; because the motion was probably caused by the breath hold (16.6 seconds) required with our scanner prototype, our second scanner prototype has been built with an acquisition time of 9 seconds and should reduce motion artifacts and increase comfort for subjects.

The most important barrier to adoption of breast CT as a general screening modality is visualization of microcalcification lesions. Adaptive noise reduction techniques, along with engineering changes that will improve the spatial resolution of the scanner prototype, are currently being developed in our laboratory to potentially address this issue. Our workstation allows adjustment of section width thickness and reformatting of images in any desired plane, so information in regard to the number and distribution of microcalcifications will be available if conspicuity can be improved.

Our study had limitations; it was a subjective, nonblinded study that relied on only one observer. However, it was intended only to provide an initial clinical evaluation of this technology. The results of our study will need to be verified with blinded receiver operating characteristic studies by using multiple radiologists. The number of women with microcalcification lesions in our study was small $(n=12)$ and may not be a representative sample. Exclusion of the four women who had technically unsatisfactory breast CT studies may have created a bias, but because the lesions could not be evaluated at breast CT, it is 
difficult to determine whether this bias would be favorable toward breast CT or against it. The current comparison is somewhat biased in favor of screen-film mammography because the subjects were selected for breast CT on the basis of the results of their standard breast imaging studies. It is likely that breast CT will help to identify some lesions that are not seen at standard imaging. In our study, there is also a training bias that is favorable toward screenfilm mammography because of the experience of the observer with that modality. There may be a scaling bias in the wording of the question in regard to the overall comfort level of the patient during breast CT.

In summary, breast CT was approximately equal to screen-film mammography for visualization of breast lesions in our selected group of women. Breast CT appears to be superior for visualization of masses, but screen-film mammography currently appears to be better for imaging microcalcification lesions. Women found breast CT to be significantly more comfortable than screen-film mammography. Breast CT is a promising technology that may have many potential applications. Further studies are needed to clarify the applications of breast CT.

Acknowledgment: We thank Laurel A. Beckett, $\mathrm{PhD}$, Department of Public Health Sciences, Division of Biostatistics, University of California, Davis, for her assistance with statistical analysis.

\section{References}

1. Tabar L, Vitak B, Chen H, et al. The Swedish
Two-County Trial twenty years later. Radiol Clin North Am 2000;38:625-651.

2. Mandelson M, Oestreicher N, Porter P, et al. Breast density as a predictor of mammographic detection: comparison of intervaland screen-detected cancers. J Natl Cancer Inst 2000;92:1081-1087.

3. Rosenberg R, Hunt W, Williamson M, et al. Effects of age, breast density, ethnicity, and estrogen replacement therapy on screening mammographic sensitivity and cancer stage at diagnosis: review of 183,134 screening mammograms in Albuquerque, New Mexico. Radiology 1998;209:511-518.

4. Pisano E, Gatsonis C, Hendrick E, et al. Diagnostic performance of digital versus film mammography for breast-cancer screening. N Engl J Med 2005;353:1773-1783.

5. Niklason L, Christian B, Niklason L, et al. Digital tomosynthesis in breast imaging. Radiology 1997;205:399-406.

6. Lehman C, Blume J, Weatherall P, et al. Screening women at high risk for breast cancer with mammography and magnetic resonance imaging. Cancer 2005;103:18981905.

7. Kriege M, Brekelmans C, Boetes C, et al. Efficacy of MRI and mammography for breast-cancer screening in women with a familial or genetic predisposition. N Engl J Med 2004;351:427-437.

8. Chang C, Sibala J, Gallagher J, et al. Computed tomography of the breast: a preliminary report. Radiology 1977;124:827-829.

9. Chang C, Sibala J, Fritz S, et al. Computed tomographic evaluation of the breast. AJR Am J Roentgenol 1978;131:459 - 464.

10. Chang C, Sibala J, Fritz S, et al. Specific value of computed tomographic breast scanner $(\mathrm{CT} / \mathrm{M})$ in diagnosis of breast diseases. Radiology 1979;132:647-652.
11. Gisvold J, Karsell P, Reese E. Clinical evaluation of computerized tomographic mam mography. Mayo Clin Proc 1977;52:181185.

12. Gisvold J, Reese D, Karsell P. Computed tomographic mammography (CTM). AJR Am J Roentgenol 1979;133:1143-1149.

13. Chen B, Ning R. Cone-beam volume CT mammography imaging: feasibility study. In: Antonuk L, Yaffe M, eds. Proceedings of SPIE: medical imaging 2001-physics of medical imaging. Vol 4320. Bellingham, Wash: International Society for Optical Engineering, 2001; 655-664.

14. Tornai M, Bowsher J, Jaszczak R, et al. Mammotomography with pinhole incomplete circular orbit SPECT. J Nucl Med 2003; 44:583-593.

15. Glick S, Vedantham S, Karellas A. Investigation of optimal $\mathrm{kVp}$ settings for CT mammography using a flat panel imager. In: Antonuk L, Yaffe M, eds. Proceedings of SPIE: medical imaging 2002-physics of medical imaging. Vol 4682. Bellingham, Wash: International Society for Optical Engineering, 2002; 392-402.

16. Boone J, Nelson T, Lindfors K, et al. Dedicated breast CT: radiation dose and image quality evaluation. Radiology 2001;221:657667.

17. Manitoba Centre for Health Policy Web site. http://www.umanitoba.ca/centres/mchp /concept/dict/ci_median/code.html. Published October 13, 2000. Accessed June 1, 2007.

18. Kuhl C, Schrading S, Leutner C, et al. Mammography, breast ultrasound, and magnetic resonance imaging for surveillance of women at high familial risk for breast cancer. J Clin Oncol 2005;23:8469-8476.

19. Lewin J, Isaacs P, Vance V, et al. Dual-energy contrast-enhanced digital subtraction mammography: feasibility. Radiology 2003; 229:261-268. 


\section{Radiology 2008}

\section{This is your reprint order form or pro forma invoice}

(Please keep a copy of this document for your records.)

Reprint order forms and purchase orders or prepayments must be received 72 hours after receipt of form either by mail or by fax at 410-820-9765. It is the policy of Cadmus Reprints to issue one invoice per order.

Please print clearly.

Author Name

Title of Article

Issue of Journal

Number of Pages

Color in Article?

Reprint \#

$\mathrm{KB} \#$

Publication Date

Symbol Radiology

Please include the journal name and reprint number or manuscript number on your purchase order or other correspondence.

Order and Shipping Information

Reprint Costs (Please see page 2 of 2 for reprint costs/fees.)

Number of reprints ordered $\$$

Number of color reprints ordered \$

Number of covers ordered

Subtotal $\$$

Taxes

$\$$

(Add appropriate sales tax for Virginia, Maryland, Pennsylvania, and the District of Columbia or Canadian GST to the reprints if your order is to be shipped to these locations.)

First address included, add \$32 for each additional shipping address

TOTAL \$
Shipping Address (cannot ship to a P.O. Box) Please Print Clearly

Name

Institution

Street

City _ State __ Zip

Country

Quantity Fax

Phone: Day

E-mail Address Evening

Additional Shipping Address* (cannot ship to a P.O. Box)

Name

Institution

Street

City

Country

Quantity

Phone: Day

E-mail Address

* Add \$32 for each additional shipping address

\section{Payment and Credit Card Details}

Enclosed: Personal Check Credit Card Payment Details

Checks must be paid in U.S. dollars and drawn on a U.S. Bank.

Credit Card: _ VISA _ Am. Exp. _ MasterCard

Card Number

Expiration Date

Signature:

Please send your order form and prepayment made payable to:

\section{Cadmus Reprints}

P.O. Box 751903

Charlotte, NC 28275-1903

Note: Do not send express packages to this location, PO Box. FEIN \#:541274108

Signature

described in this document.

\section{Invoice or Credit Card Information}

Invoice Address Please Print Clearly

Please complete Invoice address as it appears on credit card statement

Name

Institution

Department

Street

City

Country

State

Phone

E-mail Address

Cadmus will process credit cards and Cadmus Journal Services will appear on the credit card statement.

If you don't mail your order form, you may fax it to 410-820-9765 with your credit card information.

Date 


\section{Radiology 2008}

Black and White Reprint Prices

Domestic (USA only)

\begin{tabular}{|c|c|c|c|c|c|c|}
\hline $\begin{array}{c}\text { \# of } \\
\text { Pages }\end{array}$ & $\mathbf{5 0}$ & $\mathbf{1 0 0}$ & $\mathbf{2 0 0}$ & $\mathbf{3 0 0}$ & $\mathbf{4 0 0}$ & $\mathbf{5 0 0}$ \\
\hline $\mathbf{1 - 4}$ & $\$ 221$ & $\$ 233$ & $\$ 268$ & $\$ 285$ & $\$ 303$ & $\$ 323$ \\
\hline $\mathbf{5 - 8}$ & $\$ 355$ & $\$ 382$ & $\$ 432$ & $\$ 466$ & $\$ 510$ & $\$ 544$ \\
\hline $\mathbf{9 - 1 2}$ & $\$ 466$ & $\$ 513$ & $\$ 595$ & $\$ 652$ & $\$ 714$ & $\$ 775$ \\
\hline $\mathbf{1 3 - 1 6}$ & $\$ 576$ & $\$ 640$ & $\$ 749$ & $\$ 830$ & $\$ 912$ & $\$ 995$ \\
\hline $\mathbf{1 7 - 2 0}$ & $\$ 694$ & $\$ 775$ & $\$ 906$ & $\$ 1,017$ & $\$ 1,117$ & $\$ 1,220$ \\
\hline $\mathbf{2 1 - 2 4}$ & $\$ 809$ & $\$ 906$ & $\$ 1,071$ & $\$ 1,200$ & $\$ 1,321$ & $\$ 1,471$ \\
\hline $\mathbf{2 5 - 2 8}$ & $\$ 928$ & $\$ 1,041$ & $\$ 1,242$ & $\$ 1,390$ & $\$ 1,544$ & $\$ 1,688$ \\
\hline $\mathbf{2 9 - 3 2}$ & $\$ 1,042$ & $\$ 1,178$ & $\$ 1,403$ & $\$ 1,568$ & $\$ 1,751$ & $\$ 1,924$ \\
\hline Covers & $\$ 97$ & $\$ 118$ & $\$ 215$ & $\$ 323$ & $\$ 442$ & $\$ 555$ \\
\hline
\end{tabular}

\begin{tabular}{|c|c|c|c|c|c|c|}
\hline \multicolumn{7}{|c|}{ International (includes Canada and Mexico) } \\
\hline $\begin{array}{c}\text { \# of } \\
\text { Pages }\end{array}$ & $\mathbf{5 0}$ & $\mathbf{1 0 0}$ & $\mathbf{2 0 0}$ & $\mathbf{3 0 0}$ & $\mathbf{4 0 0}$ & $\mathbf{5 0 0}$ \\
\hline $\mathbf{1 - 4}$ & $\$ 272$ & $\$ 283$ & $\$ 340$ & $\$ 397$ & $\$ 446$ & $\$ 506$ \\
\hline $\mathbf{5 - 8}$ & $\$ 428$ & $\$ 455$ & $\$ 576$ & $\$ 675$ & $\$ 784$ & $\$ 884$ \\
\hline $\mathbf{9 - 1 2}$ & $\$ 580$ & $\$ 626$ & $\$ 805$ & $\$ 964$ & $\$ 1,115$ & $\$ 1,278$ \\
\hline $\mathbf{1 3 - 1 6}$ & $\$ 724$ & $\$ 786$ & $\$ 1,023$ & $\$ 1,232$ & $\$ 1,445$ & $\$ 1,652$ \\
\hline $\mathbf{1 7}-\mathbf{2 0}$ & $\$ 878$ & $\$ 958$ & $\$ 1,246$ & $\$ 1,520$ & $\$ 1,774$ & $\$ 2,030$ \\
\hline $\mathbf{2 1 - 2 4}$ & $\$ 1,022$ & $\$ 1,119$ & $\$ 1,474$ & $\$ 1,795$ & $\$ 2,108$ & $\$ 2,426$ \\
\hline $\mathbf{2 5 - 2 8}$ & $\$ 1,176$ & $\$ 1,291$ & $\$ 1,700$ & $\$ 2,070$ & $\$ 2,450$ & $\$ 2,813$ \\
\hline $\mathbf{2 9 - 3 2}$ & $\$ 1,316$ & $\$ 1,452$ & $\$ 1,936$ & $\$ 2,355$ & $\$ 2,784$ & $\$ 3,209$ \\
\hline Covers & $\$ 156$ & $\$ 176$ & $\$ 335$ & $\$ 525$ & $\$ 716$ & $\$ 905$ \\
\hline
\end{tabular}

Minimum order is 50 copies. For orders larger than 500 copies, please consult Cadmus Reprints at 800-407-9190.

\section{Reprint Cover}

Cover prices are listed above. The cover will include the publication title, article title, and author name in black.

\section{Shipping}

Shipping costs are included in the reprint prices. Domestic orders are shipped via UPS Ground service. Foreign orders are shipped via a proof of delivery air service.

\section{Multiple Shipments}

Orders can be shipped to more than one location. Please be aware that it will cost $\$ 32$ for each additional location.

\section{Delivery}

Your order will be shipped within 2 weeks of the journal print date. Allow extra time for delivery.

\section{Color Reprint Prices}

\begin{tabular}{|c|c|c|c|c|c|c|}
\hline \multicolumn{7}{|c|}{ Domestic (USA only) } \\
\hline $\begin{array}{c}\text { \# of } \\
\text { Pages }\end{array}$ & $\mathbf{5 0}$ & $\mathbf{1 0 0}$ & $\mathbf{2 0 0}$ & $\mathbf{3 0 0}$ & $\mathbf{4 0 0}$ & $\mathbf{5 0 0}$ \\
\hline $\mathbf{1 - 4}$ & $\$ 223$ & $\$ 239$ & $\$ 352$ & $\$ 473$ & $\$ 597$ & $\$ 719$ \\
\hline $\mathbf{5 - 8}$ & $\$ 349$ & $\$ 401$ & $\$ 601$ & $\$ 849$ & $\$ 1,099$ & $\$ 1,349$ \\
\hline $\mathbf{9 - 1 2}$ & $\$ 486$ & $\$ 517$ & $\$ 852$ & $\$ 1,232$ & $\$ 1,609$ & $\$ 1,992$ \\
\hline $\mathbf{1 3 - 1 6}$ & $\$ 615$ & $\$ 651$ & $\$ 1,105$ & $\$ 1,609$ & $\$ 2,117$ & $\$ 2,624$ \\
\hline $\mathbf{1 7 - 2 0}$ & $\$ 759$ & $\$ 787$ & $\$ 1,357$ & $\$ 1,997$ & $\$ 2,626$ & $\$ 3,260$ \\
\hline $\mathbf{2 1 - 2 4}$ & $\$ 897$ & $\$ 924$ & $\$ 1,611$ & $\$ 2,376$ & $\$ 3,135$ & $\$ 3,905$ \\
\hline $\mathbf{2 5 - 2 8}$ & $\$ 1,033$ & $\$ 1,071$ & $\$ 1,873$ & $\$ 2,757$ & $\$ 3,650$ & $\$ 4,536$ \\
\hline $\mathbf{2 9 - 3 2}$ & $\$ 1,175$ & $\$ 1,208$ & $\$ 2,122$ & $\$ 3,138$ & $\$ 4,162$ & $\$ 5,180$ \\
\hline Covers & $\$ 97$ & $\$ 118$ & $\$ 215$ & $\$ 323$ & $\$ 442$ & $\$ 555$ \\
\hline
\end{tabular}

\begin{tabular}{|c|c|c|c|c|c|c|}
\hline \multicolumn{7}{|c|}{ International (includes Canada and Mexico)) } \\
\hline $\begin{array}{c}\text { \# of } \\
\text { Pages }\end{array}$ & $\mathbf{5 0}$ & $\mathbf{1 0 0}$ & $\mathbf{2 0 0}$ & $\mathbf{3 0 0}$ & $\mathbf{4 0 0}$ & $\mathbf{5 0 0}$ \\
\hline $\mathbf{1 - 4}$ & $\$ 278$ & $\$ 290$ & $\$ 424$ & $\$ 586$ & $\$ 741$ & $\$ 904$ \\
\hline $\mathbf{5 - 8}$ & $\$ 429$ & $\$ 472$ & $\$ 746$ & $\$ 1,058$ & $\$ 1,374$ & $\$ 1,690$ \\
\hline $\mathbf{9 - 1 2}$ & $\$ 604$ & $\$ 629$ & $\$ 1,061$ & $\$ 1,545$ & $\$ 2,011$ & $\$ 2,494$ \\
\hline $\mathbf{1 3 - 1 6}$ & $\$ 766$ & $\$ 797$ & $\$ 1,378$ & $\$ 2,013$ & $\$ 2,647$ & $\$ 3,280$ \\
\hline $\mathbf{1 7 - 2 0}$ & $\$ 945$ & $\$ 972$ & $\$ 1,698$ & $\$ 2,499$ & $\$ 3,282$ & $\$ 4,069$ \\
\hline $\mathbf{2 1 - 2 4}$ & $\$ 1,110$ & $\$ 1,139$ & $\$ 2,015$ & $\$ 2,970$ & $\$ 3,921$ & $\$ 4,873$ \\
\hline $\mathbf{2 5 - 2 8}$ & $\$ 1,290$ & $\$ 1,321$ & $\$ 2,333$ & $\$ 3,437$ & $\$ 4,556$ & $\$ 5,661$ \\
\hline $\mathbf{2 9 - 3 2}$ & $\$ 1,455$ & $\$ 1,482$ & $\$ 2,652$ & $\$ 3,924$ & $\$ 5,193$ & $\$ 6,462$ \\
\hline Covers & $\$ 156$ & $\$ 176$ & $\$ 335$ & $\$ 525$ & $\$ 716$ & $\$ 905$ \\
\hline
\end{tabular}

\section{Tax Due}

Residents of Virginia, Maryland, Pennsylvania, and the District of Columbia are required to add the appropriate sales tax to each reprint order. For orders shipped to Canada, please add 7\% Canadian GST unless exemption is claimed.

\section{Ordering}

Reprint order forms and purchase order or prepayment is required to process your order. Please reference journal name and reprint number or manuscript number on any correspondence. You may use the reverse side of this form as a proforma invoice. Please return your order form and prepayment to:

\section{Cadmus Reprints \\ P.O. Box 751903 \\ Charlotte, NC 28275-1903}

Note: Do not send express packages to this location, PO Box. FEIN \#:541274108

Please direct all inquiries to:

Rose A. Baynard

800-407-9190 (toll free number) 410-819-3966 (direct number) 410-820-9765 (FAX number) baynardr@cadmus.com (e-mail)
Reprint Order Forms and purchase order or prepayments must be received 72 hours after receipt of form. 